\title{
Medical Students' Knowledge and Attitudes Toward Female Sex Workers and Their Occupational Risk Factors
}

\author{
Jenna T. Nakagawa, ${ }^{1}$ Muge Akpinar-Elci. ${ }^{2}$
}

\begin{abstract}
Background: The tendency for female sex workers to seek health care is highly influenced by physician attitudes and behavior. By identifying medical students' attitudes toward female sex workers and assessing their knowledge of barriers to seeking care, we can focus medical training and advocacy efforts to increase access to care and improve public health outcomes. Methods: In this cross-sectional study, medical students from various countries were invited to participate in an online survey with close-ended questions and Likert scale statements. Responses were quantified and knowledge and attitude scores were assigned based on knowledge of barriers to seeking care and agreement with positive and negative attitude statements. Results: A total of 292 medical students from 56 countries completed the survey, of whom $98.3 \%$ agreed that it will be their job to provide treatment to patients regardless of occupation. Self-identified religious students conveyed more negative attitudes toward female sex workers compared to those who did not identify themselves as religious (p<0.001). Students intending to practice in countries where prostitution is legal conveyed more positive attitudes compared to those intending to practice in countries where prostitution is illegal ( $p<0.001$ ). Conclusion: Medical students largely agreed on the importance of providing care to female sex workers as a vulnerable group. In addition to addressing knowledge gaps in medical education, more localized studies are needed to understand the religious and legal influences on attitudes toward female sex workers. Such information can help focus the efforts in both medical education and communication training to achieve the desired behavioral impacts, reconciling the future generations of health care providers with the needs of female sex workers.
\end{abstract}

Keywords: Students, Medical; Attitude of Health Personnel; Sex Workers; Social Determinants of Health; Reproductive Rights (Source: MeSH-NLM).

\section{Introduction}

In 2012, the Scelles Foundation released the first world report on sexual exploitation (Available at: http://www.fondationscelles.org/pdf/rapport_mondial/sexual_exploitation_prostitution_Fondation_Scelles.pdf, updated 2012 January, cited 2014 September 4 ) and estimated that there are approximately 42 million female sex workers (FSWs) worldwide. This figure is a likely underestimate due to the criminalization and the differing definitions of sex work that render FSWs a difficult-to-reach demographic.' Clobally, FSWs bear a higher burden of sexually transmitted diseases (STDs) and sexual abuse than do other members of the population..$^{2-6} \mathrm{~A}$ pooled analysis of articles from 2007 to 2011 revealed that in low- and middle-income countries, female sex workers were 13.5 times more likely to be living with human immunodeficiency virus (HIV), compared to other women of reproductive age.? Furthermore, sex work is often associated with sex trafficking and decreased negotiating power over the terms of sex, permitting elements of physical and emotional violence that exacerbate morbidity. ${ }^{2-6}$

A complex interplay of social, political, economic and structural obstacles serve as barriers to seeking health care for FSWs. These include social stigma, fear of criminal persecution, lack of autonomy over health-related decisions and negative experiences within the health system..$^{8-13}$ From the public health perspective, ensuring protective mechanisms for sex workers is not only an issue of human rights for vulnerable individuals, but also of controlling routes of STD transmission within the general population., ${ }^{3,14}$ Indeed, the International AIDS Society reported that an estimated $15 \%$ of HIV infections in the general adult female population could be attributed to unsafe female sex work (Available at: http://www.iasociety.org/Web/ WebContent/File/KAPs_Fact_Sheet_Sex_Workers.pdf, updated 2014 March, cited 2014 September 4).

Health care providers themselves have a significant role in ensuring health rights for their patients, potentially advocating for the provision of care to sex workers as a vulnerable population. ${ }^{15-17}$ Negative experiences with doctors-anxiety associated with physical exams, discomfort with discussing sensitive topics such as STDs, fear of judgment by medical professionals or male doctor insensitivity to female sex workers' needs-can become significant barriers to service use. ${ }^{4,10,18-21}$ These findings highlight the importance of health care provider attitudes and behavior in shaping the patient experience and, ultimately, health outcomes.

Despite the proven influence of health care providers on the utilization of care, there has been little research assessing the competence of medical students in their ability to provide for those involved with sex work. With the aim to produce competent future doctors, medical institutions have the responsibility of training students to identify socio-economic risk factors for

Submission: Jul 28, 2014

Acceptance: $\operatorname{Sep} 13,2014$

Process: Peer-reviewed

${ }^{1}$ MD/MPH Candidate 2016, Department of Public Health and Preventive Medicine, St. George's University School of Medicine, Grenada, West Indies

${ }^{2} \mathrm{MD} / \mathrm{MPH}$, Department of Public Health and Preventive Medicine, St. George's University School of Medicine, Grenada, West Indies. Center for

Global Health, Old Dominion University, Norfolk, VA.

Correspondence:

Jenna Toki Nakagawa.

Address: St. George's University, True Blue, Grenada.

Email: Jenna.Nakagawa@gmail.com 
disease, promote access to care and provide specialized care to vulnerable individuals. For that reason, knowledge and attitudes of medical students toward FSWs need to be assessed to identify any misconceptions or attitudes that may compromise care for this particular group of patients in the future. With proper training, medical students may help improve health outcomes by reducing barriers to seeking care.

The aim of this study was to identify medical students' attitudes toward female sex workers and knowledge of risk factors, such as potential barriers to seeking care, associated with the sex industry. The results would identify gaps in knowledge, attitudes and practices and underscore any common misconceptions and factors leading to negative attitudes or unwillingness to provide care. These results could be used to target specific attitudes or areas of doubt, direct the integration of social and occupational determinants of health within medical training and enable the future generations of doctors to be well-prepared advocates for FSW health and the health of the general population.

\section{Methods}

This cross-sectional study collected data for a five-month period from August 2012 to January 2013. Participation was invited through the International Federation of Medical Students' Associations (IFMSA), which is a non-governmental, international body that encompasses 108 medical student organizations across 101 different countries. The IFMSA is an appropriate source for international representation of medical students, since the web-based, transnational communication among members is frequent and reliable.

Using the IFMSA email server, all medical student members of the IFMSA were invited to participate in a web-based survey on medical student attitudes toward sex workers and knowledge of occupational risk factors associated this specific group of patients. The target population included all English-speaking IFMSA members currently enrolled in medical programs at the time of data collection. The emailed invitation letter included a link to an online survey created through SurveyMonkey.com LLC. By proceeding to the online survey, students confirmed their informed consent to participate in the study.

Knowledge and attitudes were quantified using scales similar to those used in the study of health care providers' attitudes toward sex workers by Phrasisombath, et al. ${ }^{20}$ Based on their answers to multiple-choice questions included in the survery, participants received a total knowledge score that was assessed as a raw score. To account for uncertainty and omissions, this score was also assessed as a percentage of total questions answered. Knowledge questions were based on data from previous studies on FSW health risks and care-seeking behavior. ${ }^{2-6,8-13,20-22}$ The legality of prostitution in specific countries was based on the Joint United Nations Programme on HIV/AIDS (UNAIDS) data from 2010 in an online information sheet (http:// www.unaids.org/en/media/unaids/contentassets/documents/ priorities/20100728_HR_Poster_en.pdf).

Attitudes were quantified based on a scale of $1-5$, with " 1 " corresponding to "strongly disagree" and " 5 " to "strongly agree" for positive attitude statements. The scale was reversed for negative attitude statements. Therefore, agreement with more positive attitu- des would overall approach a " 5 " score for each question, yielding a total score of 45 for the greatest agreement with positive attitude statements. With a maximum score of 45 , a score greater than or equal to 22 was interpreted as an overall "positive attitude," and a score less than 22 was interpreted as an overall "negative attitude."

Data was analyzed using StatPlus ${ }^{\circledR}$ (2009) and Microsoft Excel $\circledast$ (2011). Descriptive statistics were generated to survey attitudes toward female sex workers and assess general knowledge of potential barriers to seeking care. Linear regression was conducted to determine whether there was any correlation between knowledge and attitude scores. Regression analysis was also conducted to find any correlation between years of experience, measured by age and number of terms in medical school, and student knowledge or attitudes.

Two-sample T-tests were conducted to determine the significance of any differences in attitudes and knowledge found among the groups of students identified upon observational analysis as having disparate answers, i.e. those groups divided by sex, religiousness, legality of prostitution in country of intended practice and prior education in social determinants of health and/or occupational health. For these comparisons, knowledge was measured both by a difference in mean knowledge scores and a difference in the percentage of correctly answered knowledge questions. Attitudes were measured by a difference in mean attitude scores. For all T-tests, a significant difference was accepted if the corresponding $\mathrm{p}$-value fell below 0.05 .

Institutional Review Board approval to ensure the ethical nature of this investigation was obtained through St. George's University (Reference No. 12024).

\section{Results \\ Demographics}

A total of 292 medical students from 56 countries completed the survey (participation rate $=75.1 \%$ ). Countries that were most represented included: the United States $(n=68)$, Italy $(n=30)$, and Iran $(n=19)$ (Figure 1). Of all participating students, the median age was 23 years old, with the median medical school experience equaling six terms (three years) and a slight majority being female $(57.2 \%, n=167)$.

\section{Knowledge responses}

Regarding knowledge of FSW vulnerability and barriers to seeking care, students completed an average of 15 out of 20 questions, marking the answers to 5 out of 20 questions as "uncertain." of those questions which were completed, an average of $92 \%$ were answered correctly. For example, large majorities demonstrated the understanding that FSWs are at increased risk for morbidity associated with sexually-transmitted infections ( $89.4 \%, n=261$ ), violence $(85.3 \%, n=249)$, and drug abuse $(78.7 \%, n=230)$. The majority of the students were also aware that certain factors, such as health care provider perceptions ( $90.8 \%, n=265)$, financial burden $(90.4 \%, \mathrm{n}=264)$ and comfort with knowing test results (91.7\%, $n=268)$, are important factors in FSW decisions to seek care. With regards to knowledge about policy, $16.2 \%(n=45)$ were uncertain whether prostitution is legal in the country where they intend to practice medicine, and $27.4 \%(n=76)$ incorrectly reported that prostitution is either legal or illegal when the opposite is true. Numbers of correct responses did not vary significantly 
Figure 1. Percentages of participants from the top 14 most represented countries*

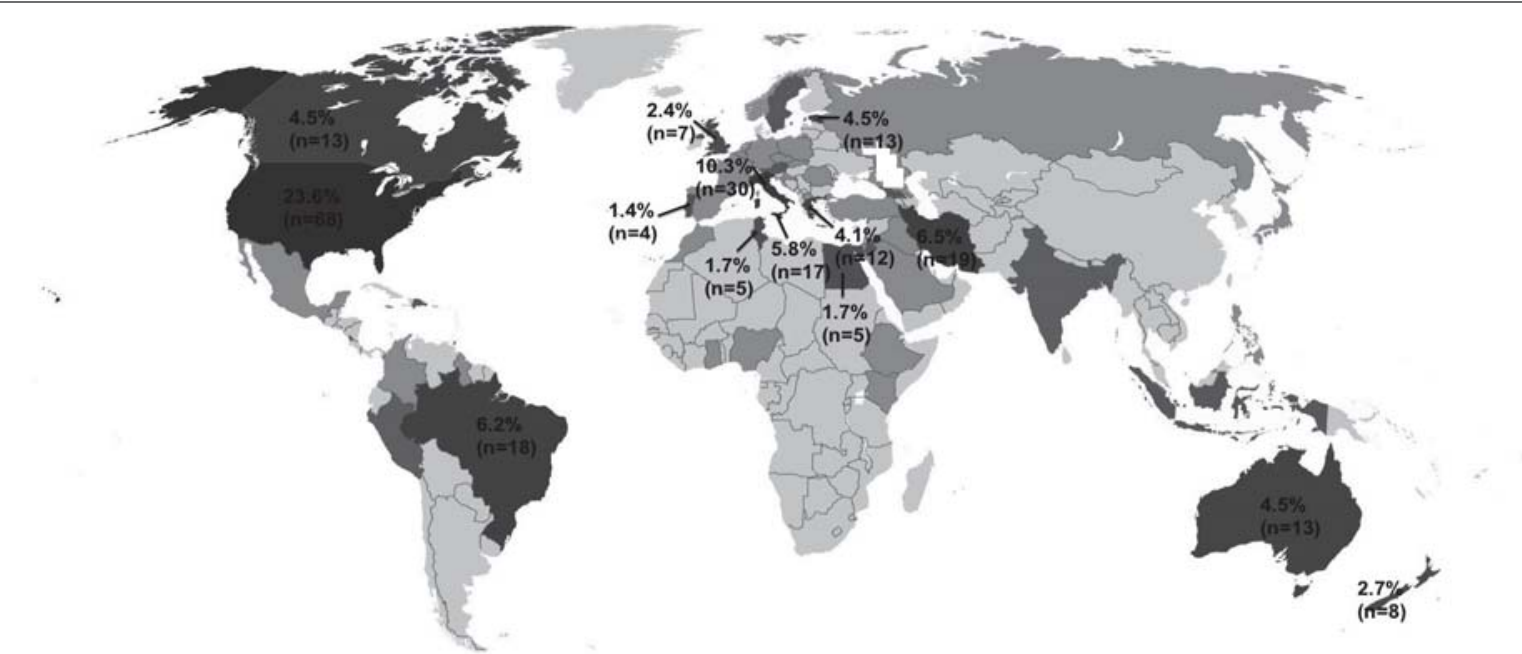

*0ther 42 participating countries contained three participants $(1 \%)$ or less of the total study sample $(n=292)$

by sex, educational background, self-identified "religiousness", country of origin or country of intended practice.

\section{Attitude statements}

Of all students, $96.5 \%(n=277)$ received a summary attitude score reflecting a positive attitude. There were large percentages of agreement with two positive-attitude statements: $98.3 \%$ $(n=297)$ of students either agreed or strongly agreed that it will be their job to provide treatment to patients regardless of occupation and $81.9 \%(n=239)$ either agreed or strongly agreed that FSWs comprise a vulnerable population that deserves particular attention. There was a prevailing agreement $(68.4 \%$, $\mathrm{n}=199$ ) with one negative-attitude statement in particular, that FSWs who do not seek care when necessary are irresponsible.

Large percentages of students were uncertain whether they agreed or disagreed with the following attitude statements: that FSWs tell the truth about symptoms of sexually transmitted infections $(51.7 \%, n=150)$, FSWs follow treatment for symptoms of sexually transmitted infections $(63.9 \%, \mathrm{n}=186)$ and that students themselves, as future doctors, will have enough time with their patients to identify victims of violence or human trafficking $(39.9 \%, \mathrm{n}=116)$.

Upon further analysis of the data by participant demographics, we found significant differences in mean attitude scores when students were divided by two criteria in particular: self-identified religiousness and the legality of prostitution in the country of intended practice. Self-identified religious students conveyed more negative attitudes compared to those who did not identify themselves as religious ( $p<0.001$ ) and students intending to practice where prostitution is legal conveyed more positive attitudes compared to those intending to practice where prostitution is illegal ( $p<0.001)$. Groups analyzed based on other variables, such as sex and prior education in social determinants of health and/or occupational health, showed no significant variation in attitudes.

\section{Relationship between knowledge and attitudes}

With regression analysis of knowledge and attitudes, we found that knowledge was positively correlated with attitudes, with higher knowledge scores of FSW risk factors and barriers to seeking care corresponding to higher (more positive) attitude sco- res $(r=0.163, p=0.006)$. There was no correlation between years of experience, measured both by age and by number of terms in medical school, and knowledge scores. In addition, there was no correlation between years of experience and attitude scores.

\section{Discussion}

Medical students from different backgrounds largely agreed on the importance of providing care to FSWs as a vulnerable group. The near-unanimous agreement across different countries, cultures, and political environments reflects the potential for the medical profession to be a nonpartisan, secular space in which patient welfare and human rights take precedent over physician biases. This support for human rights can be leveraged to strengthen equitable medical practice, focusing training and advocacy on the areas of doubt that may influence access to and quality of services. Nonetheless, the results of this study present medical education with significant challenges. In order to address gaps in education and training, we may need to focus education outside of medical institutions to include religious and political sectors that may influence medical student attitudes. Therefore, we will first explore how specific medical student competencies might be improved through formal education, followed by how concerted efforts toward health equity might be achieved through partnerships between medical education and other sectors of society.

The first area of doubt to address is the marked uncertainty regarding FSW truthfulness about symptoms and compliance with medications. While this uncertainty most likely reflects a lack of experience working with such population, it would be problematic if future treatment becomes based on assumptions about honesty and compliance rather than respectful consideration of unique patient needs. Previous studies on FSWs have shown that others' negative perceptions, including those of health care providers, are among the most important factors in influencing a FSW's decision to seek care. ${ }^{4,10,18-21}$ According to UNAIDS and other advocacy group publications, sensitivity training for medical students and physicians that emphasizes individual patient concerns and capabilities may ensure more feasible treatment options and increase the likelihood that FSW patients will return for care (Available from: http://data.unaids. 
org/publications/IRC-pub05/ic438-femsexwork_en.pdf, updated 2000 November; cited 2014 May 29). ${ }^{22}$

Secondly, the prevailing agreement that "FSWs who do not seek care when necessary are irresponsible" implies medical students' lack of awareness of multiple barriers to seeking care. Underlying this attitude is the misconception that FSWs are autonomous and can therefore seek help at will, when in fact there are many structurally-reinforced obstacles to seeking medical attention. These include the inability to leave work premises, dependence on employers for health information, financial instability and fear of criminal persecution. ${ }^{8-13,21}$ The finding that many students did not understand the legal environment of prostitution in their countries of intended practice is not surprising, considering the complex nature of legislature regarding sex work. Nonetheless, the assumption of FSW responsibility in the context of such obstacles reiterates the need for medical students to understand barriers to seeking care in order to best encourage access. While many of the obstacles exist within the term of "sex work" itself, professionals can encourage health-care seeking behavior by reassuring confidentiality and safety measures within the health care system and referring to protection programs when necessary. ${ }^{16}$

Finally, efforts must be made to address the large percentage of students who doubted they would have enough time to identify victims of violence. Such identification may not require additional time than that of a thorough history and physical exam. ${ }^{4}$ Therefore, medical institutions should continue to emphasize risk factors and warning signs associated with sex work and sex trafficking to increase the awareness of health care professionals of the at-risk individuals who may present within their patient population. 4.5

The aforementioned educational targets (dispelling prejudices to encourage patient-centered care, adequate education about obstacles to seeking care and adequate training in recognizing warning signs of abuse associated with sex work) are only preliminary components of a concerted effort to sensitize medical students to the role of physicians in promoting the health of sex workers. In the World Health Organization's Communication for Behavioral Impact (COMBI) toolkit, authors state that "Experts have come to realize that community understanding of diseases and their spread is complex, context-dependent and culturally mediated. Integration of participatory approaches in public health responses is essential to look in the right places, ask the right questions and listen more effectively before making technical recommendations and implementing interventions" (Available at: http://www.who. int/ihr/publications/combi_toolkit_outbreaks/en, updated 2012; cited 2014 May 30). Participatory approaches take into consideration the factors that influence attitudes and the matters that are deeply important to individuals in order to find feasible, mutually beneficial, sustainable behaviors. ${ }^{24}$ Medical students and physicians may deeply value acknowledgment of patient needs, adherence to personal moral values and/or promotion of social justice, all in variable orders of priority. ${ }^{25-27} \mathrm{COMBI}$ action plans, historically successful for vector-borne disease prevention (Available at: http://www.tropika.net/review/061001-Dengue_Behaviour_change/article.pdf, updated 2006; cited 2014 May 30), are also applicable for other family and community health initiatives and may improve sexual and reproductive health outcomes by reconciling health care provider interests with the health needs of female sex workers. ${ }^{28}$
Our results suggested that student attitudes were polarized mostly by personal moral beliefs (self-identified religiousness) and the legal environment (legality of prostitution in the country of intended medical practice), rather than by other variables such as age, number of years in medical school and previous education in social determinants of health or occupational health. It would be beneficial for future studies to investigate how personal moral beliefs and political environments may influence attitudes, compared to experience within the medical education system. Due to personal moral beliefs and legal environments varying significantly among societies and cultures, medical students would need to be surveyed on more local yet comprehensive scales in order to appreciate the role of specific religions on professional attitudes and legal restrictions on medical practice, medical student and physician priorities when addressing FSWs and the socioeconomic nuances that shape resource availability. Understanding these influences is the first step in developing regional COMBI action plans, leveraging medical students' desire to provide services that fit within their moral and political frameworks, while ensuring access to attentive, quality health services for FSWs.

Limitations of this study include a relatively small sample size and participation bias, with students who voluntarily participated more likely to be enthusiastic about issues of health equity. IFMSA is embedded in global health activism, so members of the IFMSA are more likely to select answers with a consciousness toward human rights. Data were also influenced by the most-represented countries' cultural perceptions of prostitution, the role of the health care professional, and rights toward reproductive health decisions, among many factors influencing attitudes. Female participation was slightly higher; however, we believe this is not a strong limitation to our study since there is no significant statistical difference in responses between males and females. For these reasons, we cannot make generalizations about the world's population of medical students. However, our results may lay a basis for future studies to investigate how religious backgrounds and surrounding policy for sex work might influence medical student knowledge, attitudes and practice toward female sex workers and, therefore, local health outcomes.

Medical students from different backgrounds largely agreed on the importance of providing care to female sex workers as a vulnerable group. However, students expressed marked uncertainty regarding FSW truthfulness and compliance as patients, demonstrated low level of awareness to FSW barriers to seeking care and uncertainty of their own competency as future health care providers. Together, these may negatively impact the quality of care. While formal training in FSW vulnerabilities and needs are a first step toward building health care personnel competencies, this study suggested that students may be more influenced by religious notions and local legislation toward sex work than education. Therefore, further studies are needed to understand the sociocultural and political influences that shape the provision of health care so that focused "communication for behavioral impact" programs can be implemented. With appeal to the widespread and non-discriminatory agreement to serve vulnerable populations, such efforts must bring access to care for FSWs to the forefront of medical-based human rights issues, empowering health care professionals to promote quality, nondiscriminatory, patient-centered care as an inalienable human right. These efforts are particularly fundamental for those at risk and necessary for improved public health in our communities. 


\section{References}

1. Vandepitte J, Lyerla R, Dallabetta G, Crabbé F, Alary M, et al. Estimates of the Number of Female Sex Workers in Different Regions of the World. Sex Transm Infect. 2006 Apr 18;82:iii18-iii25.

2. Rusch ML, Brouwer KC, Lozada R, Strathdee SA, Magis-Rodriguez C, et al. Distribution of Sexually Transmitted Diseases and Risk Factors by Work Locations Among Female Sex Workers in Tijuana, Mexico. Sex Transm Dis. 2010 0ct;37(10):608-14.

3. Wolffers I, Van Beelen N. Public Health and the Human Rights of Sex Workers. Lancet. 2003 Jun 7;361(9373):1981.

4. Baldwin S, Eisenman DP, Sayles JN, Ryan C, Chuang KS. Identification of Human Trafficking Victims in Health Care Settings. Health Hum Rights. 2011 Jul 14;13(1):1-14.

5. Dovydaitis T. Human Trafficking: The Role of the Healthcare Provider. I Midwifery Womens Health. 2011 Sep-0ct; 55(5):462-7.

6. Zimmerman C, Hossain M, Yun K, Gajdadziev V, Guzun N, et al. The Health of Trafficked Women: A Survey of Women Entering Posttrafficking Services in Europe. Am J Public Health. 2008 Jan;98(1):55-59.

7. Baral S, Beyrer C, Muessig K, Poteat T, Wirtz AL, et al. Burden of HIV Among Female Sex Workers in Low-income and Middle-income Countries: A Systematic Review and Meta-analysis. Lancet Infect Dis. 2012 Jul;12(7):538-49.

8. Ahmad K. Call for Decriminalization of Prostitution in Asia. Lancet. 2001 Aug 25;358(9282):643.

9. Cupta J, Raj A, Decker MR, Reed E, Silverman JG. HIV Vulnerabilities of Sex-trafficked Indian Women and Girls. Int J Gynaecol Obstet. 2009 0ct; $107(1): 30-4$.

10. Lieber E, Li L, Wu Z, Rotheram-Borus MJ, Guan J. HIV/STD Stigmatization Fears as Health-Seeking Barriers in China. AIDS Behav. 2006 Sep;10(5):463-71. 11. Rosenheck R, Ngilangwa D, Manongi R, Kapiga S. Treatment-seeking Behavior for Sexually Transmitted Infections in a High-risk Population. AIDS Care. 2010 Nov;22(11):1350-8.

12. Shannon K, Strathdee SA, Shoveller J, Rusch M, Kerr T, et al. Structural and Environmental Barriers to Condom Use Negotiation with Clients Among Female Sex Workers: Implications for HIV-Prevention Strategies and Policy. Am J Public Health. 2009 Apr;99(4):659-65.

13. Vijeyarasa R, Stein RA. HIV and Human Trafficking-Related Stigma: Health Interventions for Trafficked Populations. JAMA. 2010 Jul 21;304(3):344-5.

14. Wawer M], Podhisita C, Kanungsukkasem U, Pramualratana A, McNamara R. Origins and Working Conditions of Female Sex Workers in Urban Thailand: Consequences of Social Context for HIV Transmission. Soc Sci Med. 1996
Feb;42:453-62.

15. Sanders T. Protecting the Health and Safety of Female Sex Workers: The Responsibility of All. BJOG. 2007 Jul;114(7):791-3.

16. Mardh PA, Shoubnikova M, Genc M, Chaplinkas S, Unzeitig V. Health Care of Female Commercial Sex Workers. Eur J Contracept Reprod Health Care. 1999 Sep;4(3):165-80.

17. Rekart ML. Sex Work Harm Reduction. Lancet. 2005 Dec 17;336(9503):2123-34. 18. Goodyear M, Cusick L. Protection of Sex Workers. BMJ. 2007 Jan 13;334(7584):52-3.

19. Boynton P, Cusick L. Sex Workers to Pay the Price. BMJ. 2006 Jan 26;332(7535):190-1.

20. Phrasisombath K, Thomsen S, Hagberg J, Sychareun V, Faxelid E. Knowledge About Sexually Transmitted Infections and Attitudes Toward Female Sex Workers with STI Symptoms Among Health Care Providers in Laos. Asia Pac J Public Health. 2012 Nov;24(6):940-52.

21. Phrasisombath K, Thomsen S, Sychareun V, Faxelid E. Care Seeking Behaviour and Barriers to Accessing Services for Sexually Transmitted Infections Among Female Sex Workers in Laos: A Cross-sectional Study. BMC Health Services Research. 2012 Feb 14;12:37.

22. Nuttbrock LA, Rosenblum A, Magura S, Villano C, Wallace J. Linking Female Sex Workers with Substance Abuse Treatment. J Subst Abuse Treat. 2004 $0 c t ; 27(3): 233-9$.

23. Alexander P. Key Issues in Sex Work-related HIV/AIDS/STD Prevention Interventions. AIDS Health Promotion Exchange; 1992;1:4-6.

24. DiClemente RJ, Crosby RA, Kegler MC. Emerging Theories in Health Promotion, Practice and Research. John Wiley and Sons, Inc. Jossey-Bass: San Francisco. 2012.

25. Daaleman TP, Frey B. Spiritual and Religious Beliefs and Practices of Family Physicians. J Fam Pract. 1999 Feb;48(2):98-104.

26. Beach MC, Meredith LS, Halpern J, Wells KB, Ford DE. Physician Conceptions of Responsibility to Individual Patients and Distributive Justice in Health Care. Ann Fam Med. 2005 Jan-Feb;3(1):53-9.

27. Jotkowitz AB, Click S, Porath A. A Physician Charter on Medical Professionalism: A Challenge for Medical Education. Euro J Int Med. 2004 Feb; 15(1):5-9. 28. Ramalah KD, Vijay-Kumar KN, Hosein E, Krishnamoorthy P, Augustin DJ, et al. A Campaign of 'Communication for Behavioural Impact' to Improve Mass Drug Administrations Against Lymphatic Filariasis: Structure, Implementation, and Impact on People's Knowledge and Treatment Coverage. Ann Trop Med Parasitol. 2006 Jun; 100(4):345-61.

\section{Acknowledgments}

The authors would like to thank Mr. Michael Cann for his help in data analysis; the International Federation of Medical Students' Associations and all participating students for their contributions to this study.

\section{Conflict of Interest Statement a Funding}

The Authors have no funding, financial relationships or conflicts of interest to disclose. The primary investigator independently provided all financing for this study.

\section{Author Contributions}

Conception and design the work/idea: JTN. Collect data/obtaining results: JTN. Analysis and interpretation of data: JTN. Write the manuscript: JTN. Critical revision of the manuscript: JTN, MAE. Approval of the final version: JTN, MAE.

Cite as:

Nakagawa JT, Akpinar-Elci M. Medical Students' Knowledge and Attitudes Toward Female Sex Workers and Their Occupational Risk Factors. Int J Med Students. 2014 Jul-0ct;2(3):104-8. 